\title{
Clinical Applications of a Non-ablative Fractional Dual Laser (1550/1927 nm)
}

\author{
Ho Sun Chang ${ }^{1}$ \\ Nam Kyu Lim²
}

${ }^{1}$ Medicastle Clinic, Cheonan, Korea
${ }^{2}$ Department of Plastic \& Reconstructive Surgery,
Dankook University Hospital, Cheonan, Korea

Received November 29, 2020

Accepted December 18, 2020

\author{
Correspondence \\ Nam Kyu Lim \\ Department of Plastic and Reconstructive \\ Surgery, Dankook University Hospital, 201 \\ Manghyang-ro, Dongnam-gu, Cheonan 31116 , \\ Korea \\ Tel.: +82-41-550-6477 \\ Fax: +82-41-554-6477 \\ E-mail: linakyer@daum.net \\ (C) Korean Society for Laser Medicine and Surgery \\ (c) This is an open access article distributed under the \\ terms of the Creative Commons Attribution Non- \\ Commercial License (http://creativecommons.org/ \\ licenses/by-nc/4.0) which permits unrestricted non- \\ commercial use, distribution, and reproduction in any \\ medium, provided the original work is properly cited.
}

\begin{abstract}
The non-ablative fractional dual laser is equipped with two types of lasers, $1550 \mathrm{~nm}$ and $1927 \mathrm{~nm}$ in one device, and was approved by the United States Food and Drug Administration in 2013. The advantages of the non-ablative fractional laser (NAFL) include fewer side effects such as erythema, edema, post-laser pigmentation, and scab formation. Thus, the NAFL is preferred by both practitioners and consumers because it is convenient and safe for use. The $1550 \mathrm{~nm}$ erbium glass and $1927 \mathrm{~nm}$ thulium lasers are representative NAFLs that have been developed separately and are often used as a single-wavelength laser with proven clinical efficacy in various indications. The $1550 \mathrm{~nm}$ wavelength laser penetrates the dermis layer and the $1927 \mathrm{~nm}$ wavelength laser is effective for epidermal lesions. Therefore, targeting the skin layer can be easily achieved with both the 1550 and 1927 nm lasers, respectively, or in combination. Clinically, the $1550 \mathrm{~nm}$ laser is effective in the treatment of mild to moderate sagging and wrinkles, scars, and resurfacing. The 1927 $\mathrm{nm}$ laser improves skin texture and treats skin pigmentation and wounds. It can also be used for drug delivery. The selection and utilization rate of NAFL has been increasing in recent times, due to changes in lifestyle patterns and the need for beauty treatments with fewer side effects and short downtime. In this study, we present a plan for safe and effective laser therapy through a review of literature. Clinical applications of the multifunctional NAFL are also described.
\end{abstract}

\section{Key words}

Nonablative laser Treatment; Rejuvenation; Acne; Melasma 


\section{INTRODUCTION}

Since the concept of 'fractional thermolysis' was first introduced by Huzaira et al. in 2003 and the first fractional thermolysis device was made in 2004, various models of fractional laser have been steadily developed in the clinical field of skin rejuvenation. ${ }^{1}$ The Fraxel ${ }^{\circledR}$ laser (Solta Medical, Hayward, CA, USA) has evolved into one of the most well-known fractional lasers in the masses along with various models of several wavelengths, of which the Fraxel Dual ${ }^{\circledR}$ laser is the latest model. The Fraxel Dual ${ }^{\circledR}$ laser (Solta Medical) is a nonablative fractional laser (NAFL) developed by mounting lasers of two wavelengths, $1550 \mathrm{~nm}$ and $1927 \mathrm{~nm}$ in a single device, and had received the United States Food and Drug Administration (FDA) approval in 2013 (Table 1). ${ }^{2}$

Compared to ablative fractional laser (AFL), NAFL creates a microthermal treatment zone (MTZ) without removing the epidermis and provides a therapeutic effect in a non-peeling manner. The advantages of the NAFL include the rare formation of the scabs, few incidences of erythema and edema, short duration, low post-laser pigmentation, and rare permanent side effects. So, it is preferred both for practitioners and consumers because of its convenience and safety. ${ }^{3-5}$ The $1550 \mathrm{~nm}$ erbium glass (Er:glass) and $1927 \mathrm{~nm}$ thulium laser are representative NAFLs that have been developed separately and are often used as single-wavelength laser and have been proven to have clinical efficacy in various indications. While the 1550 $\mathrm{nm}$ wavelength can penetrate the dermis layer, the 1927 $\mathrm{nm}$ wavelength is suitable for the epidermal lesion. So, targeted skin layer can be easily achieved with both 1550 $\mathrm{nm}$ and $1927 \mathrm{~nm}$ respectively, or in combination. ${ }^{2}$ The dual laser, which incorporates these two wavelengths in one device, can be seen as a device that maximizes the advantages of the nonablative fractional treatment method. Clinically, the $1550 \mathrm{~nm}$ laser can be applied to the treatment of various skin problems such as mild to moderate sagging, scars, rejuvenation of aging skin, and

Table 1. FDA-approved area of Fraxel Dual ${ }^{\circledR}$ laser (http://fda.gov)

\begin{tabular}{cc}
\hline Wavelength & FDA approved Clinical Indication (2013) \\
\hline $1550 \mathrm{~nm}$ laser & $\begin{array}{c}\text { Skin resurfacing, soft tissue coagulation procedure, } \\
\text { treatment of dyschromia and cutaneous diseases } \\
\text { (such as lentigos, actinic keratosis, and melasma) } \\
\text { treatment of periorbital wrinkles, acne scars and } \\
\text { surgical scars }\end{array}$ \\
$1927 \mathrm{~nm}$ laser & $\begin{array}{c}\text { Soft tissue coagulation, actinic keratosis, treatment of } \\
\text { pigmented lesions (such as lentigos, freckle) }\end{array}$ \\
\hline
\end{tabular}

FDA, the United States Food and Drug Administration. acne vulgaris. The $1927 \mathrm{~nm}$ laser improves skin texture and treats skin pigmentation, drug delivery, and superficial wounds. ${ }^{2}$

However, it is a well-established theory that AFL is superior to NAFL in terms of clinical efficacy. It is undeniable that repeated NAFL treatments are necessary to attain the effects of a single treatment of AFL. ${ }^{6}$ Nevertheless, the frequency of difficult complications such as postinflammatory pigmentation (PIH) and permanent scars which are especially common in oriental and female skin, is extremely low after NAFL treatment rather than AFL. The selection and utilization rate of NAFL is higher in modern society, considering the lifestyle patterns and treatment needs of beauty treatment patients who prefer fewer side effects. This tendency is expected to further expand in the field of skin laser treatment. ${ }^{7-9}$ Therefore, the consideration of the clinical application of NAFL would be meaningful.

\section{CLINICAL APPLICATION}

\section{Skin rejuvenation}

Like all the organs in our body, the skin also ages based on age favorites. As an internal factor of skin aging, the main cause is the shortening of telomeres at gene terminals and changes in hormones, and ultraviolet (UV) rays are the most important cause as an external factor. Smoking, stress, malnutrition, and pollutants have been also demonstrated to external factors in skin aging. Under such condition, reactive oxygen species (ROS) are generated, thereby promoting cell aging. ${ }^{10,11}$ Skin aging induces changes in the pre-structured layers of the face. ${ }^{12}$ Aged lor photoaged) skin shows macroscopic wrinkles, pigmentation, skin atrophy, and loss of organized epidermal and dermal architecture, flattening of rete ridge, cutaneous thinning with reducing collagen fiber in histologically. With aging, collagen content in the dermis decreases by $1 \%$ every year, and it has been reported that the decrease is particularly clear with about $20 \%$ in photoaged areas until the age of 80 years. In addition to the reduction in collagen produced by senescent fibroblasts, glycosaminoglycan is also reduced, and senile fibroblasts also exhibit a late response to growth factors. Interestingly, when measuring the procollagen gene expression in aged skin, collagen loss is most pronounced in the upper third of the dermis, which is probably associated with UV skin penetration. ${ }^{11}$

Both ablative and nonablative fractional laser are effective for dermis remodeling and show similar histological findings and changes in the molecular mediator. ${ }^{3}$ Ablative laser resurfacing has been known as the most effective 
method for treating wrinkles, and atrophic scars, but it sometimes has long recovery periods after treatment with side effects such as erythema, pigmentation, and high frequency of herpes infections. ${ }^{13}$ With the development of fractional photothermolysis methods that can reduce the downtime and side effects, there have been attempts to replace the fractional technology as alternative and the use of NAFL is increasing. One of the representative NAFL is the $1550 \mathrm{~nm}$ Er:glass laser. ${ }^{14}$ Although ablative laser resurfacing is gold standard treatment in severe photoaged skin, 8,9 the NAFL showed the similar clinical results in mild to moderate cases. Moreover, it showed an increase in the success rate of treatment when used in combination with treatments such as botulinum toxin and filler. ${ }^{14,15}$

A single one-time NAFL treatment has little impressive improvement effect, but it does not interfere with daily life. As the treatment has only a few inconveniences and side effects, it is easy to repeat the treatment and can be used in combination with other devices. This usefulness of the therapy opens up the possibility of clinical use of NAFL for the treatment of mild and moderate skin aging. In addition, in patients with Fitzpatrick skin types IV to VI, the $1550 \mathrm{~nm}$ Er:glass laser reduced the frequency of side effects. ${ }^{16}$ This report is thought to help in laser treatment of oriental people who are fragile to side effects such as post-inflammatory hyperpigmentation (PIH) associated with laser. In our cases, patients with Fitzpatrick type IIIIV can experience fewer side effects and recurrences during nonablative fractional dual laser treatment (Fig. 1). Side effects after laser are usually slight and temporary. According to the literature investigated by Fraxel Dual ${ }^{\circledR}$ Laser, about $5 \%$ of treated patients showed side effects; $1.8 \%$ of prolonged erythema, PIH (1.1\%), erythema exacerbation $(0.9 \%)$, herpes recurrence $(0.6 \%)$, and acne recurrence $(0.2 \%)$. But long-term severe adverse reactions were not reported. ${ }^{17,18}$

We recommend considering two treatment strategic models at the time of designing treatment methods and setting parameters according to the purpose of treat-
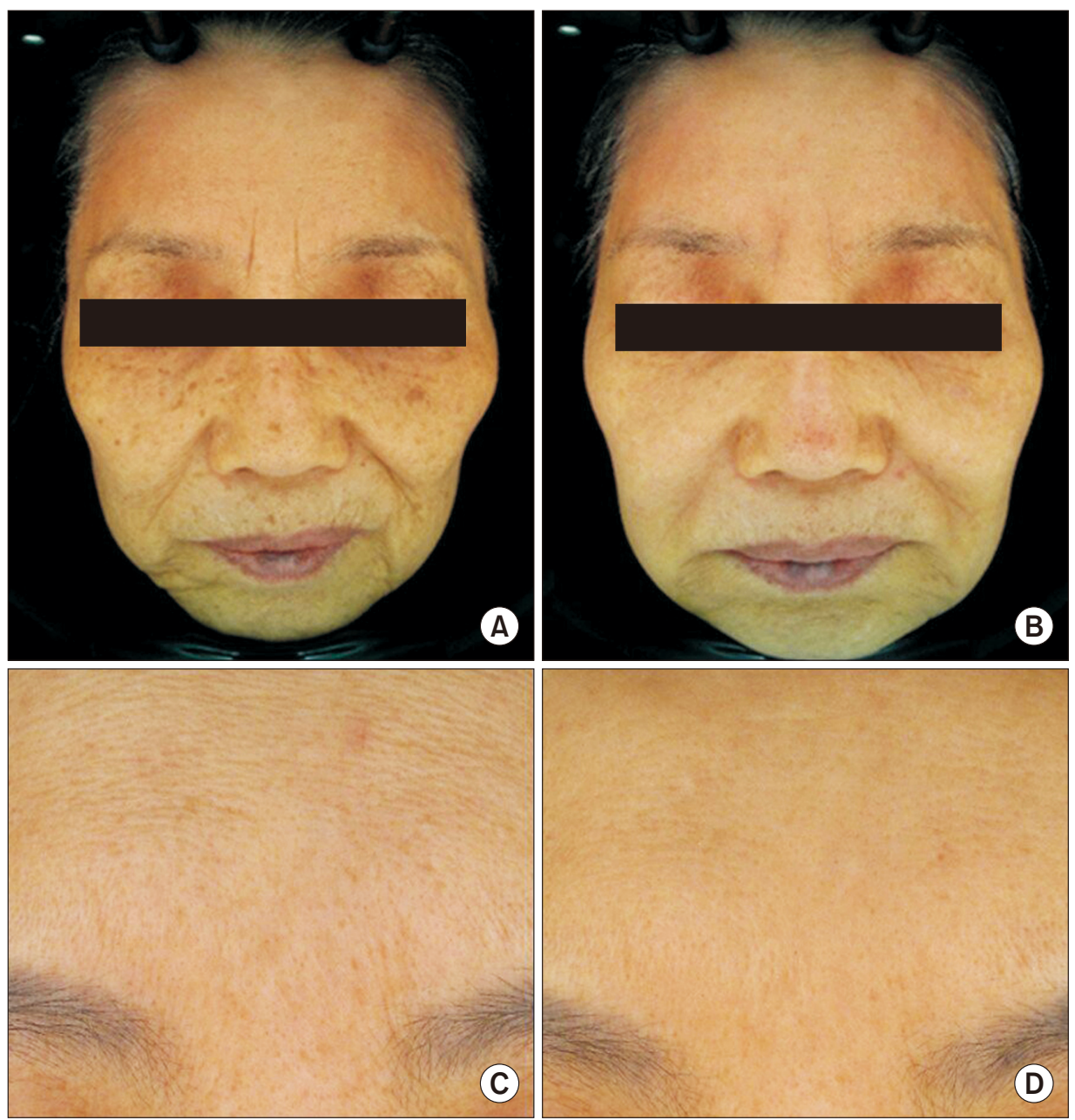

Fig. 1. Skin rejuvenation cases treated with nonablative fractional dual laser. (A) Preclinical photograph. (B) Clinical photograph after treatment. Improvement in the wrinkles on the entire face can be seen. (C) Preclinical photograph. (D) Clinical photograph after treatment. Improvement in the wrinkles on the forehead can be seen. 
ment. One strategy is inducing heat energy to wrinkles directly with high energy. And the other one is indirect heating transmission with medium to low energy overlapping and channeling which can be effectively performed to increase the penetration of the drug and active ingredient (Fig. 2). In the $1550 \mathrm{~nm}$ Er:glass laser, the primary chromophore is water in the tissue, the absorption into hemoglobin becomes much higher compared to the lasers with a shorter wavelength band of $1320 \mathrm{~nm}$ and $1440 \mathrm{~nm}$. So, the light and heat effect can be well-transmitted into the dermis in a relatively short time. After four treatments at 6-week intervals, dermis thickness has been reported to increase by approximately $17 \%$, and the collagen synthesis were not shown difference significantly despite of the energy level (15 mJ vs. $70 \mathrm{~mJ}$ ). Therefore, lower microbeam energy and high microbeam density are important to achieve optimal treatment. ${ }^{13}$

However, post-laser pigmentation studies suggest a slightly different effect, which affects beam density more than energy. The report showed that increasing the number of treatments with low density improved clinical efficacy and reduced PIH (from 18.2 to 6\%). ${ }^{15.16}$ In other words, we propose that a strategy of increasing microbeam density to increase collagen synthesis, whereas increasing the number of treatments with low density nesting is effective in pigmentation treatments.

The $1927 \mathrm{~nm}$ thulium laser demonstrated effective results in terms of improvement in pigmented lesions and resurfacing of photoaged skin even in the relatively low energy of 5-10 mJ. ${ }^{18}$ Nonfractional ablative dual laser for skin rejuvenation is a new single or combination therapy that reduces side effects, discomfort, duration of illness, and provides safe and effective therapeutic effects in a



Fig. 2. Schematic diagram of two treatment strategies for nonablative fractional dual laser. PDT, photodynamic therapy. way that enables the continuation of daily life without any problems.

\section{Acne scar}

Acne is a chronic inflammatory skin disease that $80 \%$ of adolescents and young adults experience, with repeated inflammation and secondary complications such as pigmentation, scarring, and erythema, along with cosmetic problems and the development of low self-esteem. It has been reported that there exists a possibility of social and psychological effects such as emotional difficulty, social isolation, anger, and depression. ${ }^{19}$ Acne is caused by multiple factors: 1) sebum produced by sebaceous gland, 2) formation of sebum colony pocket of Cutibacterium acnes, 3) change in keratinization process, and 4) inflammatory-mediated secretion. ${ }^{20}$ The first-line treatments for acne are conventionally topical and oral medications, but the side effects and contraindications of the medications, which often do not provide complete improvement, can be significant, and the frequent recurrence of acne is clinically proven. In such cases, the alternative treatment should be needed. ${ }^{20}$

The Er:glass laser reacts with water-containing epidermal keratinocytes, collagen, blood vessels, and other skin tissues, and partially damages the sebum. In general, the wavelength band that exhibits the optimal photothermal effect of the sebaceous glands is $1210-1760 \mathrm{~nm}$, and the $1550 \mathrm{~nm}$ and $1540 \mathrm{~nm}$ Er:glass lasers are absorbed by the sebaceous gland and surrounding dermal matrix. ${ }^{15,21}$ The depth of the sebaceous gland is 200-1000 $\mu \mathrm{m}$, and to treat acne, it is necessary to penetrate to a depth of about $400 \mu \mathrm{m}$, which can cause heat damage to the sebaceous gland and follicular infundibulum. The principle of treating acne using the Er:glass laser can be divided into two parts. One method is that the heat causes directly injury to the sebaceous gland and infundibulum directly, and the other one is drug delivery with photosensitivity agent. 22,23,25 Looking at the studies on short-term and medium- to long-term effects for the treatment of acne using the $1540 \mathrm{~nm}$ or $1550 \mathrm{~nm}$ Er:glass laser. Bogle et al. treated patients with moderate to severe inflammatory facial acne four times at two-week intervals. Six months after treatment (10 J/cm², $4-6$ pulses), $68 \%$ of patients and $78 \%$ of physicians reported improvement. ${ }^{22}$ In another study, $1550 \mathrm{~nm}$ laser was followed up every 3 months for 1 year after 4 treatments (30-40 $\mathrm{mJ}$ ) at 2-week intervals, thereby resulting in a significant reduction in the number of acne lesions and the size of sebaceous gland. ${ }^{23}$ According to the medium- to long-term study, follow-up results of $1550 \mathrm{~nm}$ laser after 4 treatments (169 spot density and 
$15-30 \mathrm{~mJ} / \mathrm{cm}^{2}$ ) at 4-week intervals, showed improvement of $72 \%$ in 6 months and $79 \%$ in 1 year. The side effects were temporary erythema and edema, and some patients showed recurrence of acne and increased skin sensitization, but all the patients had reduced sebuminduced skin slippage..$^{21}$ In another long-term study using a $1540 \mathrm{~nm}$ laser for facial acne, $71 \%$ improvement was reported after 6 months after 4 treatments (20\% nest, 40 $\mathrm{J} / \mathrm{cm}^{2}$ ) at 4-week intervals and $79 \%$ per year. The subjects responded well to various acne lesions such as nodules, comedones, papules, and pustules with reduced sebuminduced skin slippage. ${ }^{24}$ In summary, it was observed that the therapeutic effect of Er:glass laser is excellent, and the effect maintained for a relatively long period of time without remarkable side effects. Considering the burden of long-term oral peels prescription, the NAFL is good option as alternative treatment for active acne vulgaris and depressed acne scar together.

In the following method, the energy absorption is increased when a photosensitive agent is applied during the operation of Er:glass laser. Studies have shown that applying 7.5\% aminolevulinic acid (ALA) after $1550 \mathrm{~nm}$ laser treatment increased the absorption of photosensitive agents depending on the laser energy and operation time..$^{25}$ By taking advantage of this point, it is possible to treat acne scars and sebaceous gland at the same time to reduce the onset of acne as well as to improve the wound. So, two treatment objectives can be achieved, thereby leading to the patient's satisfaction. We believe that it is possible to improve the degree and therapeutic effect in a short period of time and propose the method as an effective strategy.

The optimal standard for scar treatment is known as the ablative laser. However, due to the long recovery time, discomfort, and several side effects that occur during large-scale laser peeling, fractional type of ablative laser using $\mathrm{CO}_{2}$ and Er:YAG are often used. ${ }^{1,8}$ Even with $A F L$, however, at least a week time is required for the scab to fall off spontaneously, which can interfere with daily life. Considering the side effects such as deposition and erythema, NAFL can be an alternative method. A comparative study of the facial acne scars shows that the AFL has a better improvement effect than the NAFL (AFL 26-83\% vs. NAFL 26-50\%). However, the duration of erythema (AFL 3-14 days vs. NAFL 1 day), the incidence of PIH (AFL 92.3\% vs. NAFL 13\%), the pain score (AFL 5.9-8.1 vs. NAFL 3.9-5.6) provide a good idea about the benefits of NAFL treatment. ${ }^{1}$ If so, when is the optimal timing to manage scars with the NAFL treatment? Concerning thyroid surgery with a $1550 \mathrm{~nm}$ laser, a study revealed that treating the wound 3 weeks after surgery was significantly better than the group that started the treatment 3 months and 6 months after surgery. ${ }^{26}$ In the future, it is thought that numerous attempts will be made to start relatively early scar treatment using the NAFL after surgery. Based on this theory, satisfactory results were obtained that the acne scar was treated with a nonablative fractional dual laser (Fig. 3).

\section{Melasma}

How can the $1550 \mathrm{~nm}$ or $1927 \mathrm{~nm}$ laser, of which chromophore is water, help treat pigmented diseases and melasma? The mechanism of treatment for pigmented diseases by NAFL can be summarized as follows. 1) Emission of melanin by the 'melanin shuttle' mechanism, 2) Increased selective penetration of whitening agents, 3) Recovery of the dermis environment by an increase in fibroblasts and inhibition of physiological melanin action. All the effects can be observed simultaneously. ${ }^{18,27}$

The process of the melanin shuttle is as follows. The microscopic thermal zones (MTZ) generated by fractionated photothermolysis also affect keratinocytes and collagen fibers, thereby forming cylindrical necrosis of
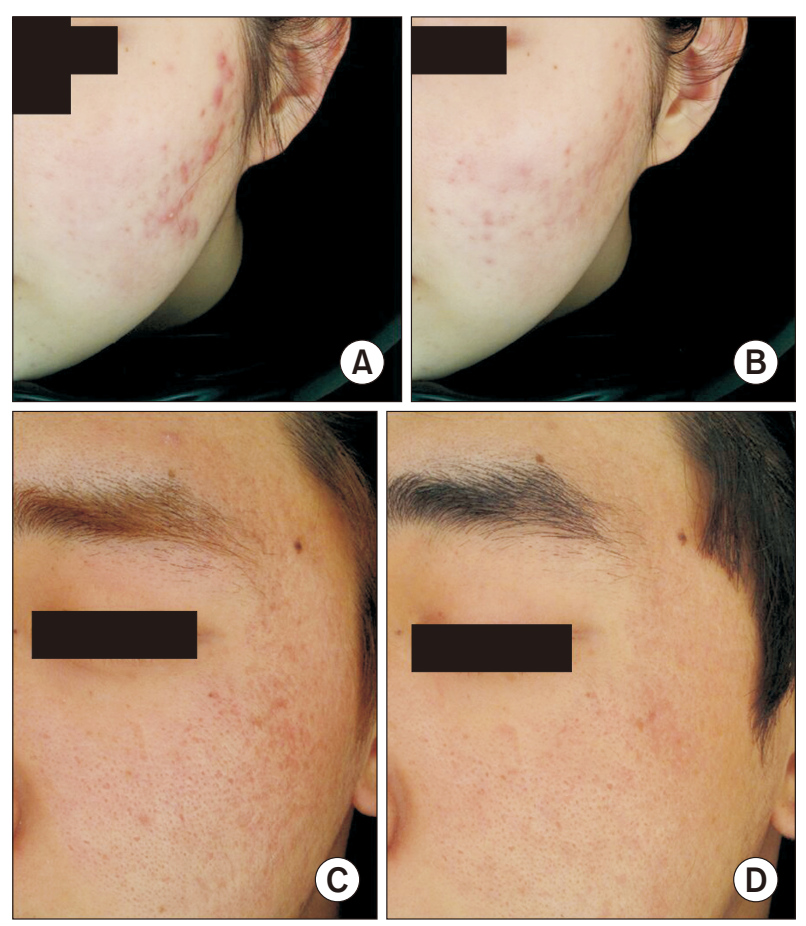

Fig. 3. Acne scar cases treated with nonablative fractional dual laser. (A) Preclinical photograph. (B) Clinical photograph after treatment. Acute acne can be resolved after treatment. (C) Preclinical photograph. (D) Clinical photograph after treatment. Improvement of the chronic acne scars can be seen. 

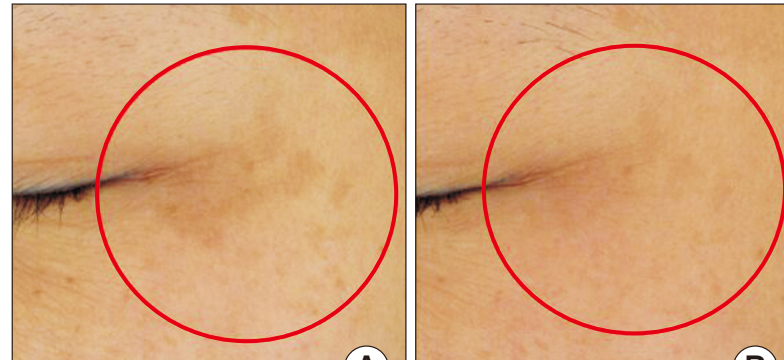

(A)


Fig. 4. Melasma case treated with nonablative fractional dual laser. (A) Preclinical photograph. (B) Clinical photograph after treatment. Improvement in the melasma around the left eye can be seen. (C) Preclinical photograph (polarized). (D) Clinical photograph after treatment (polarized). Improvement in the melasma around the left eye can be seen.

intraepidermal keratinocytes called microscopic epidermal necrotic debris (MENDs). Keratinocytes and dermal tissue move towards the stratum corneum and are removed. The mechanism by which NAFL removes melanin from the dermis and epidermis is called 'Melanin Shuttles'. ${ }^{28}$ Also, as with the AFL, application of drugs and active ingredients after NAFL treatment increases delivery, and application of a whitening agent after selective laser treatment of pigmented lesions increases the whitening effect on the target area. ${ }^{29}$ Nowadays, a focus of melasma and pigmented diseases has been changed to the surrounding skin environment from a melanocytecentric perspective. Melanocytes are affected by cell-tocell interaction with keratinocytes or dermal fibroblasts, and studies have been established that involve genes, paracrine factors, and receptors, but they are still unclear. The process of melanogenesis and migration is affected by various external stimuli and the nervous and hormonal systems. The melanocytes at the cellular level are affected by the reaction of cytokines and receptors secreted by keratinocytes and fibroblasts (paracrine factors). They are affected by a dual inhibitory effect with regulatory proteins of gene expression. ${ }^{30}$ In the Wnt receptor of melanocytes, the intracellular reaction related to melanin synthesis is suppressed by Wnt inhibitory factor (WIF), and when WIF is suppressed or blocked (double suppression), melanin synthesis is conversely increased. ${ }^{31}$ Looking at the effects of pigments on factors derived from fibroblasts in the dermis, the action is like a double-edged sword. For example, Dickkopf 1 (DKK1) and neuregulin-1 are reported to be associated with physiologic pigmentation, and stem cell factor (SCF), hepatocyte growth factor (HGF), and basic fibroblast growth (bFGF) are reported as arguments associated with pathologic pigmentation. Especially, the SCF secreted from fibroblasts is known as a typical aggravating factor for melasma. DKK1 secreted from fibroblasts in the dermis has been reported to suppress the Wnt signal pathway. Compared to the torso, palm and sole showed hypopigmented state. Some researchers explained its phenomenon due to the Wnt inhibitory effect of DKK1. ${ }^{32}$ Also, the WIF-1 is a typical tumor suppressor that has various effects on the body, and it suppresses cancer and skin pigments. ${ }^{33}$ In melasma lesions, the WIF1 which a suppressor of canonical and noncanonical Wnt signaling, appears to be down-regulated. Decreased WIF1 from fibroblasts and even from keratinocytes are known to significantly stimulate the production of melanin and the transmission of some melanocytes to form melasma. In one of WIF studies, regulation of Wnt and DKK signaling pathways is usually observed in pigmented disorders, they suggested that the regulation of Wnt inhibitory factors and DDK pathways in the dermis and epidermis would be effective to treat melasma. ${ }^{31,34}$ From this point of view, it is interesting to note how the $1550 \mathrm{~nm}$ laser treatment affects fibroblasts. It is believed that laser inducedactivated fibroblasts could affect surrounding fibroblasts by signal transmission. This concept can be used to treat pigmented diseases, and if research on the exact conditions comes out, it will be of great help in the treatment.

From a clinical point of view, various attempts and studies have been continued to treat pigmented diseases and melasma in Asian skin. Unlike white people, they have potential complications such as post-laser induced PIH or hypopigmentation, and recurrence. To overcome these limitations, various studies have been attempted to treat melasma with low energy and density of NAFL treatment. In studies used to treat melasma with the $1550 \mathrm{~nm}$ laser alone, the results showed temporary improvement and then the possibility of recurrence exacerbation emerged. For example, in a study conducted with $1550 \mathrm{~nm}$ laser alone $\left(6-10 \mathrm{~mJ}, 2000-2500 \mathrm{MTZ} / \mathrm{cm}^{2}, 4\right.$ treatments at 2-week intervals) in patients with Fitzpatrick Scale III / IV, melanocyte count and peripheral keratinocytes reported a decrease in melanin content and patients with 
Fitzpatrick Scale III / IV stains Treatment) after $1550 \mathrm{~nm}$ laser treatment (6-12 mJ, 1-2 week intervals, 4-6 times). ${ }^{27}$ However in another report, at 12-week follow-up, patient ratings were $75-100 \%$ and physician ratings were $50-60 \%$ improvement, while the initial 1-2 treatment results were better than the result of medium- to long-term follow up. ${ }^{35}$ In another study, a division comparing the $1064 \mathrm{~nm}$ Q-switched Nd:YAG (1064 QSNY) single treatment and the merged treatment (1064 QSNY laser plus $1550 \mathrm{~nm}$ laser) at Fitzpatrick skin type III / IV melasma patients, this study results revealed a significant improvement in each group, but no significant difference between the two groups could be demonstrated at 4 and 12 weeks after the last treatment. ${ }^{36}$

The $1927 \mathrm{~nm}$ laser, which attracted attention in the treatment of pigmented diseases, also showed good effects at the early stages, but recurrence and rebound phenomenon were observed. Treatment of melasma with $1927 \mathrm{~nm}$ laser improved the Melasma Area and Severity Index (MASI) score by $28-45 \%$, but the effect diminished 2 months after treatment and a recurrence was reported at 3 months. ${ }^{37}$ The effects of the single NAFL treatment of melasma showed various and temporary outcomes in either the $1550 \mathrm{~nm}$ or $1927 \mathrm{~nm}$ lasers. Thus, increasing the number of treatments with low-density and low-energy is a key of reducing the side effects to treat pigmented diseases. Long-term planned research is demanded to increase the efficacy of NAFL in field of melasma treatment. ${ }^{37}$

Meanwhile, the laser merge therapy has been proposed to overcome the above-mentioned problem. A recent combination therapy study reported treatment of patients with Fitzpatrick skin types II-IV with a combination of 595 pulse dye laser and $1927 \mathrm{~nm}$ laser. Its result showed that $54 \%$ of patients improved their melasma and the efficacy was prolonged until 3 months without complications. ${ }^{38}$ Also another merge therapy with topical tranexamic acid delivery, a repeat every 3 months treatment was safe and effective in long-term follow up. ${ }^{39}$

Summarizing the melasma treatments using NAFL, it is helpful to treat melasma, but further studies are considered necessary which improve the medium- to longterm effects as well as combination therapy, especially according to the skin characteristics of Asians.

\section{CONCLUSION}

In the present study, the literature and actual clinical cases of the features and clinical applications of the nonablative fractional dual laser, which is a multi-laser were examined. The fact that it is possible to return to a quick daily life and that there are relatively few sequelae are advantages for busy modern people. There are certainly limits to monotherapy, but there is no doubt that it is a good laser that can provide great satisfaction to patients when used accurately and effectively in case of a variety of lesions. We hope that this article will be useful for safe and effective laser treatment.

\section{CONFLICT OF INTEREST}

No potential conflict of interest relevant to this article was reported.

\section{REFERENCES}

1. Ong MW, Bashir SJ. Fractional laser resurfacing for acne scars: a review. Br J Dermatol 2012;166:1160-9.

2. Finney R, Torbeck R, Saedi N. Non-ablative fractional resurfacing in the treatment of scar contracture. Lasers Surg Med 2016;48:170-3.

3. Wat $\mathrm{H}$, Wu DC, Chan $\mathrm{HH}$. Fractional resurfacing in the Asian patient: current state of the art. Lasers Surg Med 2017;49:4559.

4. Taudorf EH, Danielsen PL, Paulsen IF, Togsverd-Bo K, Dierickx C, Paasch U, et al. Non-ablative fractional laser provides long-term improvement of mature burn scars--a randomized controlled trial with histological assessment. Lasers Surg Med 2015;47:141-7.

5. Park KY, Ko EJ, Seo SJ, Hong CK. Comparison of fractional, nonablative, 1550-nm laser and 595-nm pulsed dye laser for the treatment of facial erythema resulting from acne: a splitface, evaluator-blinded, randomized pilot study. J Cosmet Laser Ther 2014;16:120-3.

6. Lipozenčić J, Mokos ZB. Will nonablative rejuvenation replace ablative lasers? Facts and controversies. Clin Dermatol 2013;31:718-24.

7. Monstrey S, Middelkoop E, Vranckx JJ, Bassetto F, Ziegler UE, Meaume $S$, et al. Updated scar management practical guidelines: non-invasive and invasive measures. J Plast Reconstr Aesthet Surg 2014;67:1017-25.

8. Carniol PJ, Hamilton MM, Carniol ET. Current status of fractional laser resurfacing. JAMA Facial Plast Surg 2015;17:360-6.

9. Kim DW, Hwang NH, Yoon ES, Dhong ES, Park SH. Outcomes of ablative fractional laser scar treatment. J Plast Surg Hand Surg 2015;49:88-94.

10. Rinnerthaler M, Bischof J, Streubel MK, Trost A, Richter K. Oxidative stress in aging human skin. Biomolecules 2015;5:54589.

11. Lephart ED. Skin aging and oxidative stress: equol's anti-aging 
effects via biochemical and molecular mechanisms. Ageing Res Rev 2016;31:36-54.

12. Farage MA, Miller KW, Elsner P, Maibach HI. Intrinsic and extrinsic factors in skin ageing: a review. Int J Cosmet Sci 2008;30:87-95.

13. Alexiades-Armenakas MR, Dover JS, Arndt KA. The spectrum of laser skin resurfacing: nonablative, fractional, and ablative laser resurfacing. J Am Acad Dermatol 2008;58:719-37; quiz 738-40.

14. Sachdeva S. Nonablative fractional laser resurfacing in Asian skin--a review. J Cosmet Dermatol 2010;9:307-12.

15. Alajlan AM, Alsuwaidan SN. Acne scars in ethnic skin treated with both non-ablative fractional $1,550 \mathrm{~nm}$ and ablative fractional CO2 lasers: comparative retrospective analysis with recommended guidelines. Lasers Surg Med 2011;43:787-91.

16. Chua SH, Ang P, Khoo LS, Goh CL. Nonablative infrared skin tightening in Type IV to V Asian skin: a prospective clinical study. Dermatol Surg 2007;33:146-51.

17. Clark CM, Silverberg JI, Alexis AF. A retrospective chart review to assess the safety of nonablative fractional laser resurfacing in Fitzpatrick skin types IV to VI. J Drugs Dermatol 2013;12:42831.

18. Brauer JA, McDaniel DH, Bloom BS, Reddy KK, Bernstein LJ, Geronemus RG. Nonablative 1927 nm fractional resurfacing for the treatment of facial photopigmentation. J Drugs Dermatol 2014;13:1317-22.

19. Cohen BE, Brauer JA, Geronemus RG. Acne scarring: a review of available therapeutic lasers. Lasers Surg Med 2016;48:95115.

20. Rivera AE. Acne scarring: a review and current treatment modalities. J Am Acad Dermatol 2008;59:659-76.

21. Liu Y, Zeng W, Hu D, Jha S, Ge Q, Geng S, et al. The long-term effect of $1550 \mathrm{~nm}$ erbium:glass fractional laser in acne vulgaris. Lasers Med Sci 2016;31:453-7.

22. Bogle MA, Dover JS, Arndt KA, Mordon S. Evaluation of the 1,540-nm erbium:glass laser in the treatment of inflammatory facial acne. Dermatol Surg 2007;33:810-7.

23. Moneib H, Tawfik AA, Youssef SS, Fawzy MM. Randomized split-face controlled study to evaluate $1550-\mathrm{nm}$ fractionated erbium glass laser for treatment of acne vulgaris--an image analysis evaluation. Dermatol Surg 2014;40:1191-200.

24. Angel S, Boineau D, Dahan S, Mordon S. Treatment of active acne with an er:glass (1.54 microm) laser: a 2-year follow-up study. J Cosmet Laser Ther 2006;8:171-6.

25. Lim HK, Jeong KH, Kim NI, Shin MK. Nonablative fractional laser as a tool to facilitate skin penetration of 5-aminolaevulinic acid with minimal skin disruption: a preliminary study. Br J Dermatol 2014;170:1336-40.

26. Park KY, Oh IY, Seo SJ, Kang KH, Park SJ. Appropriate timing for thyroidectomy scar treatment using a 1,550-nm fractional erbium-glass laser. Dermatol Surg 2013;39:1827-34.

27. Lee HS, Won CH, Lee DH, An JS, Chang HW, Lee JH, et al. Treatment of melasma in Asian skin using a fractional 1,550-nm laser: an open clinical study. Dermatol Surg 2009;35:1499-504.

28. Kwon SH, Na JI, Choi JY, Park KC. Melasma: updates and perspectives. Exp Dermatol 2019;28:704-8.

29. Rongsaard N, Rummaneethorn P. Comparison of a fractional bipolar radiofrequency device and a fractional erbium-doped glass 1,550-nm device for the treatment of atrophic acne scars: a randomized split-face clinical study. Dermatol Surg 2014:40:14-21.

30. Hasegawa J, Goto Y, Murata H, Takata M, Saida T, Imokawa G. Downregulated melanogenic paracrine cytokine linkages in hypopigmented palmoplantar skin. Pigment Cell Melanoma Res 2008;21:687-99.

31. Kim JY, Lee TR, Lee AY. Reduced WIF-1 expression stimulates skin hyperpigmentation in patients with melasma. J Invest Dermatol 2013;133:191-200.

32. Yamaguchi Y, Morita A, Maeda A, Hearing VJ. Regulation of skin pigmentation and thickness by Dickkopf 1 (DKK1). J Investig Dermatol Symp Proc 2009;14:73-5.

33. Hwang I, Park JH, Park HS, Choi KA, Seol KC, Oh SI, et al. Neural stem cells inhibit melanin production by activation of Wnt inhibitors. J Dermatol Sci 2013;72:274-83.

34. Wang X, Zhu Y, Sun C, Wang T, Shen Y, Cai W, et al. Feedback activation of basic fibroblast growth factor signaling via the Wnt/ $\beta$-catenin pathway in skin fibroblasts. Front Pharmacol 2017;8:32.

35. Rokhsar CK, Fitzpatrick RE. The treatment of melasma with fractional photothermolysis: a pilot study. Dermatol Surg 2005;31:1645-50.

36. Kim HS, Kim EK, Jung KE, Park YM, Kim HO, Lee JY. A splitface comparison of low-fluence $Q$-switched Nd: YAG laser plus $1550 \mathrm{~nm}$ fractional photothermolysis vs. Q-switched Nd: YAG monotherapy for facial melasma in Asian skin. J Cosmet Laser Ther 2013;15:143-9.

37. Brauer JA, Alabdulrazzaq H, Bae YS, Geronemus RG. Evaluation of a low energy, low density, non-ablative fractional 1927 $\mathrm{nm}$ wavelength laser for facial skin resurfacing. J Drugs Dermatol 2015;14:1262-7.

38. Chan CS, Saedi N, Mickle C, Dover JS. Combined treatment for facial rejuvenation using an optimized pulsed light source followed by a fractional non-ablative laser. Lasers Surg Med 2013:45:405-9.

39. Wanitphakdeedecha R, Sy-Alvarado F, Patthamalai P, Techapichetvanich T, Eimpunth S, Manuskiatti W. The efficacy in treatment of facial melasma with thulium 1927-nm fractional laserassisted topical tranexamic acid delivery: a split-face, doubleblind, randomized controlled pilot study. Lasers Med Sci 2020;35:2015-21. 
How to cite this article: Chang HS, Lim NK. Clinical applications of a Non-ablative Fractional Dual Laser (1550/1927

nm). Med Lasers 2020;9:110-118. https://doi.org/10.25289/

ML.2020.9.2.110 